PREPARED FOR THE U.S. DEPARTMENT OF ENERGY, UNDER CONTRACT DE-AC02-76CH03073

PPPL-3690

PPPL-3690

UC-70

Understanding of Neutral Gas Transport in the Alcator C-Mod Tokamak Divertor

by

D.P. Stotler, C.S. Pitcher, C.J. Boswell, B. LaBombard, J.L. Terry, J.D. Elder, and S. Lisgo

May 2002

NM|

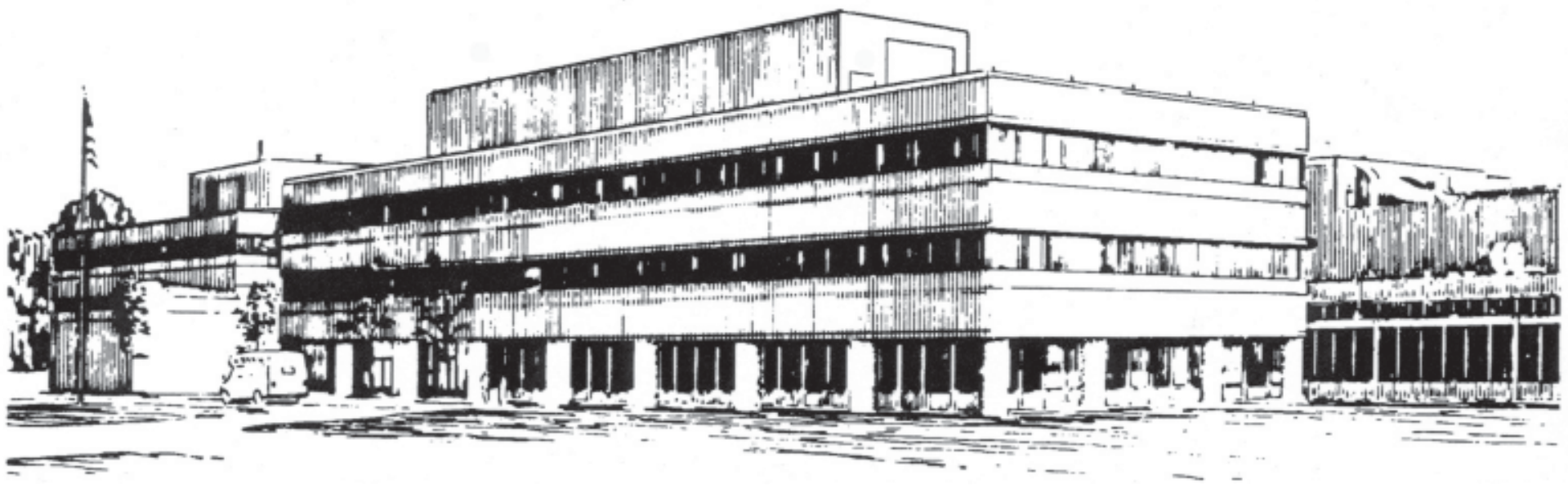

PRINCETON PLASMA PHYSICS LABORATORY PRINCETON UNIVERSITY, PRINCETON, NEW JERSEY 


\section{PPPL Reports Disclaimer}

This report was prepared as an account of work sponsored by an agency of the United States Government. Neither the United States Government nor any agency thereof, nor any of their employees, makes any warranty, express or implied, or assumes any legal liability or responsibility for the accuracy, completeness, or usefulness of any information, apparatus, product, or process disclosed, or represents that its use would not infringe privately owned rights. Reference herein to any specific commercial product, process, or service by trade name, trademark, manufacturer, or otherwise, does not necessarily constitute or imply its endorsement, recommendation, or favoring by the United States Government or any agency thereof. The views and opinions of authors expressed herein do not necessarily state or reflect those of the United States Government or any agency thereof.

\section{Availability}

This report is posted on the U.S. Department of Energy's Princeton Plasma Physics Laboratory Publications and Reports web site in Fiscal Year 2002. The home page for PPPL Reports and Publications is: http://www.pppl.gov/pub_report/

DOE and DOE Contractors can obtain copies of this report from:

U.S. Department of Energy

Office of Scientific and Technical Information

DOE Technical Information Services (DTIS)

P.O. Box 62

Oak Ridge, TN 37831

Telephone: (865) 576-8401

Fax: (865) 576-5728

Email: reports@adonis.osti.gov

This report is available to the general public from:

National Technical Information Service

U.S. Department of Commerce

5285 Port Royal Road

Springfield, VA 22161

Telephone: 1-800-553-6847 or

(703) 605-6000

Fax: (703) 321-8547

Internet: http://www.ntis.gov/ordering.htm 


\title{
Understanding of Neutral Gas Transport in the Alcator C-Mod Tokamak Divertor
}

\author{
D. P. Stotler*, C. S. Pitcher ${ }^{\dagger}$, C. J. Boswell ${ }^{\dagger}$, B. LaBombard ${ }^{\dagger}$, J. L. Terry ${ }^{\dagger}$, \\ J. D. Elder** and S. Lisgo** \\ * Princeton Plasma Physics Laboratory, Princeton University, P. O. Box 451, Princeton, NJ \\ 08543-0451, USA \\ ${ }^{\dagger}$ MIT Plasma Science and Fusion Center, NW17, Cambridge, MA 02139, USA \\ ** University of Toronto Institute for Aerospace Studies, 4925 Dufferin St., Toronto M3H 5T6,
}

Canada

\begin{abstract}
A series of experiments on the effect of divertor baffling on the Alcator C-Mod tokamak provides stringent tests on models of neutral gas transport in and around the divertor region. One attractive feature of these experiments is that a trial description of the background plasma can be constructed from experimental measurements using a simple model, allowing the neutral gas transport to be studied with a stand-alone code. The neutral-ion and neutral-neutral elastic scattering processes recently added to the DEGAS 2 Monte Carlo neutral transport code permit the neutral gas flow rates between the divertor and main chamber to be simulated more realistically than before. Nonetheless, the simulated neutral pressures are too low and the deuterium Balmer- $\alpha$ emission profiles differ qualitatively from those measured, indicating an incomplete understanding of the physical processes involved in the experiment. Some potential explanations are examined and opportunities for future exploration are highlighted. Improvements to atomic and surface physics data and models will play a role in the latter.
\end{abstract}

\section{INTRODUCTION}

The development of models for plasma transport and plasma-material interactions in the periphery of magnetically confined fusion devices aids in our understanding of the underlying physical processes and should lead to tools facilitating the design of future experiments [1]. An integral component of these models is a description of the behavior of neutral atoms and molecules generated by plasma-material interactions and volume recombination processes. Mean free paths for such atoms and molecules can be large relative to the plasma and device scale lengths, requiring a kinetic description of their transport. Furthermore, the physics and geometric detail needed for the desired solution accuracy vastly exceed that achievable by analytic means. Instead, the Monte Carlo techniques developed for simulating neutron transport have been adapted and extended [2] to fill this need. Monte Carlo codes have the advantage of permitting the incorporation of arbitrarily complicated geometry $[3,4]$ and physics into the simulations. The limit on the realism of these simulations usually hinges on the correctness of the physics involved.

The physics input to these codes can be broken down into plasma profiles, sources of neutrals, and atomic and surface physics models. The first two items, which include the electron and ion density, temperature, and flow velocity, can be obtained from analytic 
expressions, edge plasma transport codes, or models based on direct experimental measurements. We focus on the last approach since it seems to be the least uncertain given the difficulty of solving the plasma transport equations in realistic situations. Issues of diagnostic interpretation do exist [5], but lie beyond the scope of this paper.

We will describe simulations of specific experiments on the Alcator C-Mod tokamak that test the atomic and surface physics models and data used in the Monte Carlo neutral transport code DEGAS 2 [6] and illustrate how those physical processes directly impact the observable quantities. The results of the simulations differ significantly from the corresponding experimental values. We will examine potential explanations for the discrepancies by determining the sensitivity of the simulated values to changes in the assumptions underlying the simulations.

We conclude that no single satisfactory explanation can be found within the conventional model of scrape-off layer plasma transport. More detailed and realistic descriptions of the atomic and surface physics processes occurring in magnetic fusion devices may lead to improved agreement with the experimental measurements or at least eliminate some of the existing uncertainties. However, a true resolution will likely require advances in plasma transport models.

\section{ALCATOR C-MOD EXPERIMENTS}

A series of experiments on the Alcator C-Mod tokamak has directly addressed the impact of neutral gas flows between the divertor region and the main chamber (Fig. 1) on the plasma behavior. Prior to these experiments, the C-Mod device had been considered to effectively baffle neutral hydrogen and impurity species generated in the divertor so that they cannot easily make their way into the main chamber, penetrate into the confined plasma and lead to a deterioration of its pressure through radiative cooling or through effects on plasma microinstabilities.

A set of bypass valves has been installed on C-Mod to permit the neutral conductance between the divertor and main chamber to be essentially doubled in as little as 20 ms. With the bypass valves closed, diagnostic openings provide intrinsic pathways for neutrals to reach the main chamber. A principal result of these experiments is that opening the bypass reduces the divertor neutral pressure by a factor of two [7]. The current flowing through the bypass from the divertor to the main chamber is thus inferred to remain constant. Even more surprisingly, the plasma parameters, Balmer- $\alpha$ emissions, and several global characteristics of the plasma do not change substantially either.

The conclusion drawn is significant and astonishing: the Alcator C-Mod divertor operates as if it were unbaffled [7]. Neutral transport simulations with DEGAS 2 undertaken to aid in the understanding of these experiments [4] supported a hypothesized explanation based on a one-dimensional model [8]. Subsequent experiments and analysis [9] suggest that we still do not have a complete understanding of these results.

For the purposes of this paper, the key point of [4] is that the absolute values of the simulated neutral pressure are an order of magnitude too low. The particular deuterium discharge upon which the simulations are based had a line average electron density $\bar{n}_{e}=1.46 \times 10^{20} \mathrm{~m}^{-3}$. With the bypass closed (open), the pressure in the divertor plenum 


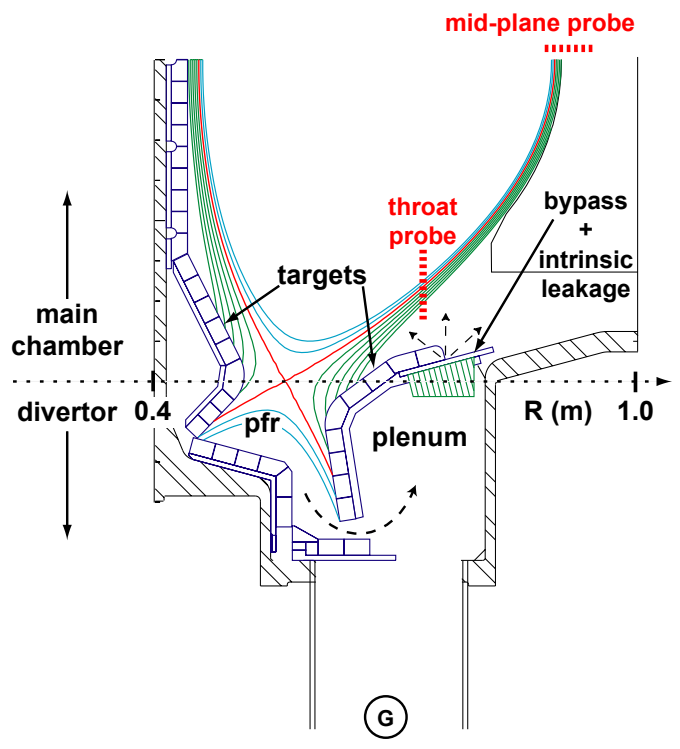

FIGURE 1. Poloidal cross section of lower half of Alcator C-Mod tokamak; $R$ is the major radius. Plasma flows radially from closed flux surfaces across the separatrix and onto the open field lines. The "X-point" of the separatrix serves as a dividing line between the "main chamber" and "divertor" portions of the vacuum vessel. The divertor "targets" are the material surfaces first intersected by these open field lines. The flux surfaces below the X-point are referred to as the "private flux region" (pfr). Neutral atoms and molecules generated by plasma-material interactions are predominantly ionized by the plasma. Some find their way through gaps (dashed curves) in the vacuum vessel into the divertor "plenum" and ducts extending downward. The neutral pressure is measured by gauge "G". Intrinsic leakage pathways plus the bypass valves permit some neutrals to reach the main chamber from the plenum. Plasma parameters are measured by two upstream reciprocating probes and by Langmuir probes embedded in the targets.

measured by an absolute capacitance gauge (Fig. 1) was 30 (15) mTorr. The other key observable is the deuterium Balmer- $\alpha\left(D_{\alpha}\right)$ emission pattern in the divertor region viewed by a radial array of downward looking detectors.

The extensive diagnostic set present on Alcator C-Mod permits the plasma parameters to be specified almost entirely by experimental measurements. The plasma conditions across the open flux surfaces adjacent to the core plasma ("upstream") are obtained from fast-scanning Langmuir-Mach probes at midplane and above the entrance to the divertor ("throat"). Fixed Langmuir probes in the target provide the plasma densities and temperatures there as well as the ion fluxes striking the target. Because the discharge density is well below the detachment density limit for the outer scrape-off layer, a simple "two point" model $[4,10]$ is expected to suffice for specifying the spatial variation of the plasma parameters between the probe locations. An ad hoc prescription for the plasma parameter variation through the private flux region was made [4] for the initial simulations. A more refined approach incorporating additional diagnostic data [11] will be discussed later in the paper.

The original simulations postulated neutral sources in the main chamber and exponential spatial decays for the plasma parameters away from the contiguous flux surfaces [4]. However, these details are not critical to this paper and have not been incorporated into the simulations described here. 


\section{DESCRIPTION OF SIMULATION AND BASELINE RESULTS}

The geometry used in DEGAS 2 is built up from a simple outline of the tokamak vacuum vessel and a magnetic equilibrium computed for the C-Mod shot and time of interest [4]. The geometry and simulations are toroidally symmetric. While this is a reasonable, and typical, approximation for the plasma behavior, the vacuum vessel structure through which the neutral gas flows possesses significant non-axisymmetric features [5]. The implications of our approximation will be discussed at the end of the paper.

Two principal sources of neutrals enter into the simulations. The first is recycling of plasma fluxes striking the divertor target plates. Physically, incoming plasma ions are accelerated toward the targets through a narrow potential that naturally forms to slow the more rapid flow of electrons towards the surface, resulting in no net current to the surface. The ions are neutralized by the electron cloud near the surface prior to coming in contact with surface atoms. In DEGAS 2, an ion is sampled from a Maxwellian distribution at the local ion temperature and drift velocity. A value for the sheath potential is computed using one of a few different, simple models. The result is an increment, typically a few times the local electron temperature, to the ion's energy representing its acceleration through the sheath.

Specific models describing the interactions of neutral atoms, such as those arising from incident ions, and molecules with material surfaces are incorporated into DEGAS 2. In general, the output of these models is a characterization of one or more produc$\mathrm{t}$ velocity distributions and values for the probability of each. In particular, the binary collision code TRIM [12] provides probabilities for reflection of deuterium atoms on molybdenum (all surfaces in these simulations) as a function of incident energy and angle. The TRIM data also contain probability distributions characterizing the outgoing atom's energy and direction relative to the incident direction. Typical reflection coefficients are between 0.5 and 0.6 ; reflected atoms usually leave the surface with a significant fraction of their incident energy.

The magnitude of tokamak plasma fluxes is large enough that the near-surface layers of the targets are quickly saturated. Typically, the plasma density reaches a steady state without additional fueling [7]. The simulation assumes that the number of incident ions and atoms per second not reflected (i.e., are absorbed) is balanced by an equal rate of molecular desorption. These deuterium molecules have an energy characteristic of the wall temperature, taken to be $300 \mathrm{~K}$. Their outgoing angular distribution is preferentially normal to the surface, but isotropic in azimuthal angle. All incident molecules are assumed to be absorbed, but likewise balanced by an equal rate of molecular desorption (i.e., no reflection).

The second neutral source in the simulations is volume recombination of deuterium ions and electrons. The recombination rate is obtained from a collisional-radiative model $[13,14]$ that also provides the rate for multi-step electron impact ionization of deuterium atoms. The collision cross-sections used in the model have been taken from [15]. The collisional-radiative model assumes that the divertor plasma is optically thin; we will address this issue later in the paper. The rates and kinetic treatment of molecular dissociation and ionization are described in [16].

Scattering of deuterium atoms and molecules off deuterium ions is effected with differential cross-sections calculated using state-of-the-art quantum mechanical techniques 


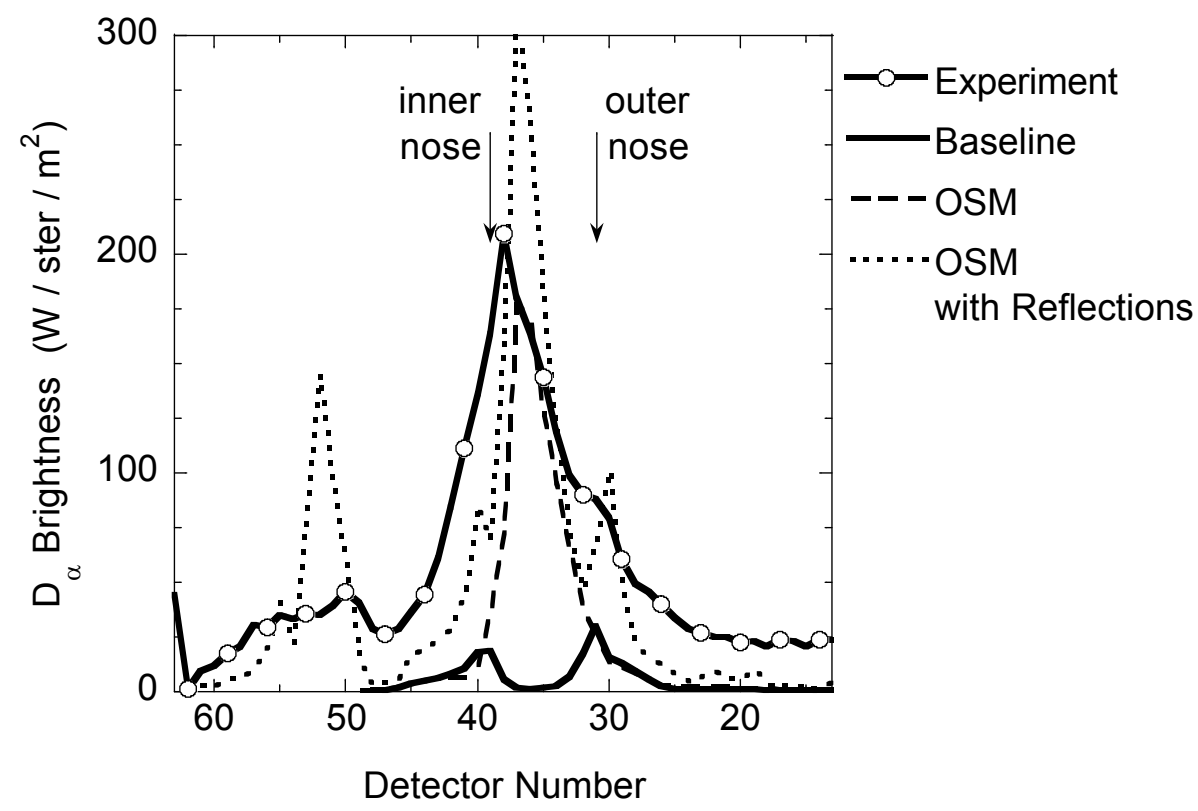

FIGURE 2. Comparison of experimental data from the array of downward looking $\mathrm{D}_{\alpha}$ detectors with several simulations.

[17]. The atom - ion interaction incorporates both classically identifiable charge exchange and elastic scattering channels. For computational efficiency, a minimum scattering angle is enforced with a constraint that the momentum transport cross-section be unaltered [18]. The differential scattering is handled using cumulative probability tables for the cosine of the scattering angle.

A simple, iterative BGK treatment of neutral-neutral elastic scattering is used $[18,19]$. For the observed pressures, the ratio of the neutral-neutral mean free path relative to a typical scale length can be as low as $\sim 0.01$ for molecules and $>1$ for atoms. The transitional conditions occurring in the problem demand a nonlinear kinetic treatment similar to the one being used here.

The baseline simulations corresponding to the bypass open and closed states yield molecular pressures near the location of the gauge of 0.74 and $0.97 \mathrm{mTorr}$, respectively. Both results are more than an order of magnitude smaller than the measured values of 15 and 30 mTorr. Likewise, the baseline simulated $\mathrm{D}_{\alpha}$ signal is everywhere well below the experimental curve (Fig. 2). Furthermore, the radial variation of the two curves is qualitatively different.

The trend of the neutral pressure and flow rate through the bypass as the bypass conductance is varied [4] are consistent with Pitcher's analytic model [8]. However, the magnitude of the pressure and $\mathrm{D}_{\alpha}$ disagreement detracts from the credibility of the simulations as a comprehensive picture of the experimental behavior. Furthermore, they indicate a serious shortcoming or omission in some aspect of the neutral transport model (including, possibly, the input experimental data). Such under-predictions of the neutral pressure are not new $[5,20,21]$. We will now examine possible explanations for the current discrepancies. 


\section{ASSESSMENT OF MODEL COMPONENTS}

Both the neutral pressure and the overall magnitude of the $\mathrm{D}_{\alpha}$ emission profile scale directly with the strength of the neutral sources [4]. The $\mathrm{D}_{\alpha}$ signal is generally indicative of a nearby source of neutral atoms. The light can be the result of a cascade down from the continuum (recombination) or due to excitation of atoms.

The two principal characteristics of the recycling source are the total current of particles reaching the divertor targets and the reflected fraction. For a given recycling current, the neutral pressure and $\mathrm{D}_{\alpha}$ signals are sensitive to the plasma model used to interpolate between the Langmuir probe data. For example, a somewhat ad hoc lowering of the plasma density and temperature immediately in front of the target effectively increases the recycling source by reducing the fraction of recycled atoms that are ionized just as they come off the surface [4].

The rest of the connection between the recycling source and the neutral pressure measured some distance, perhaps meters, away is governed by the elastic interactions between the plasma ions, recycled atoms and molecules. Recycled ions reflected as atoms will have energies comparable to the local electron temperature (a few $\mathrm{eV}$ or larger). Atoms resulting from dissociation of molecules have Frank-Condon energies of $2-3 \mathrm{eV}$. In the plasma region, the atoms are also tightly coupled to the local ion population through elastic scattering, i.e., charge exchange. The result is an atom temperature in the divertor of roughly $2 \mathrm{eV}$. On the other hand, molecules start off at the wall temperature, equivalent to an energy of only $0.03 \mathrm{eV}$.

Elastic scattering transfers momentum from the plasma ions and warm atoms to the molecules, raising their pressure [8]. Turning off the elastic scattering processes between the molecules and other species in one sensitivity test resulted in more than a factor of two decrease in the total neutral pressure [4]. Their addition represents a significant improvement in the realism of the code's atomic physics [22]. The data underlying these processes and their implementation in DEGAS 2 have now been thoroughly validated $[14,18]$. No further enhancements or modifications capable of explaining the neutral pressure discrepancy are foreseen.

The surface reflection coefficient determines the fraction of incident ions that are reflected and, hence, the balance between relatively fast atoms and slow molecules in the stream of recycled neutral particles. This balance in turn has an influence on the neutral pressure through the momentum exchanges mediated by the elastic scattering processes. Pitcher's semi-analytic model of the divertor bypass experiments produced molecular pressures of $>10$ mTorr [8]. The biggest contributor to the apparent discrepancy with the complete DEGAS 2 runs is the semi-analytic model's simplified treatment of the interactions of the neutral species with material surfaces. We can emulate that treatment in DEGAS 2 by specularly reflecting all ions / atoms incident on the divertor targets. All other surfaces are taken to yield only desorbed molecules. The result is an increase of the neutral pressure from 0.97 mTorr to 1.72 mTorr. The $\mathrm{D}_{\alpha}$ emission profile for this run does not differ significantly from the baseline curve in Fig. 2.

This sensitivity suggests taking a closer examination of the surface physics models used in DEGAS 2. However, because the material conditions found inside an operating tokamak are difficult to duplicate in the laboratory and because diagnosing tokamak plasma-material interaction in situ is even more problematic, improvements to the ide- 
alized descriptions currently used in the simulations will be slow in coming.

The source rate for recombination is determined by the plasma parameters input to DEGAS 2 via the collisional-radiative model for hydrogen. In the baseline simulation, the total source of recombined atoms is $1 \%$ of the ion flux to the targets. The resulting $\mathrm{D}_{\alpha}$ emission profile peaks near the divertor targets, whereas the experimental signal possesses a single broad peak centered about the private flux region (Fig. 2). This discrepancy suggests a much stronger recombination source in the private flux region than is provided in the baseline model.

Tomographic reconstruction of the $\mathrm{D}_{\gamma}$ emission [23] also indicates much more recombination than is predicted with the simple plasma model used to set up the input to DEGAS 2. The $\mathrm{D}_{\gamma}$ emission peaks in the private flux region between the inner target and the X-point. Ad hoc manipulations of the private flux region plasma intended to replicate the peak $\mathrm{D}_{\gamma}$ emission result in a centrally peaked $\mathrm{D}_{\alpha}$ emission pattern and an increase in the neutral pressure to 1.88 mTorr [4].

The Onion Skin Method (OSM) code [24] provides a more physically accurate approach to this task, as well as to estimating the plasma parameters between the probe locations in the main scrape-off layer [11]. The OSM model solves the plasma conservation equations in one dimension along field lines using the available experimental data as constraints. In the main scrape-off layer, the plasma sources due to neutral species are self-consistently computed with the EIRENE Monte Carlo neutral transport code. In the private flux region, the plasma description contains a number of free parameters that can be varied to yield an optimal match between the simulated and experimental $\mathrm{D}_{\alpha}$ and $\mathrm{D}_{\gamma}$ profiles. The effect of Lyman- $\alpha$ radiation trapping on the recombination rate is estimated using the EIRENE-computed neutral density to determine the local photon mean free path. The resulting plasma densities and temperatures are then cross-checked with Stark broadening measurements. The result is again a complete, two-dimensional description of the plasma density and temperature built up from the available diagnostic data with a minimal number of assumptions.

Using this plasma as input to DEGAS 2 yields a neutral pressure of 2.02 mTorr and an improvement in the comparison with the experimental $\mathrm{D}_{\alpha}$ emission pattern (Fig. 2). Despite the increased sophistication of the plasma model, the neutral pressure is again too low and the $\mathrm{D}_{\alpha}$ profile still differs significantly from the one observed experimentally. Note that the DEGAS 2 results match those produced by EIRENE as part of the OSM solution procedure. The independent simulations with DEGAS 2 not only provide confirmation of the EIRENE results, but serve as useful comparisons with the other DEGAS 2 work described in this paper.

Two physical effects missing from this DEGAS 2 calculation are radiation trapping and light reflections off of the metal surfaces inside Alcator C-Mod. For typical Alcator C-Mod divertor parameters, Lyman- $\alpha$ and $\beta$ are trapped [25]; Balmer- $\alpha$ is optically thin. Incorporating these effects into the code would lead to changes in recombination (similar to those in OSM-EIRENE) and ionization rates as well as to the ionization balance. In the DEGAS 2 simulations, the most significant impact of radiation trapping would be an increase in the ionization rate, leading to a reduction in the neutral pressure below that of the baseline simulation. Radiation trapping should be self-consistently incorporated into these simulations, although doing so may not improve the comparison with experimental data. An escape factor formalism [25] is feasible, but represents only a 
rough approximation. Some attempts at more sophisticated treatments have been made $[25,26]$. A comprehensive, but practical, model for including radiation transport into divertor plasma and neutral transport is needed.

The second missing physical effect is the reflection of the $\mathrm{D}_{\alpha}$ light off of the shiny molybdenum tiles in the Alcator C-Mod divertor. Such reflections complicate interpretation of the divertor-viewing chords. The reflectivity of molybdenum is roughly $50 \%$ at the $6560 \AA \mathrm{D}_{\alpha}$ wavelength. To assess the potential importance of this effect, we extend the integration chords used in DEGAS 2 to simulate the divertor-viewing diagnostic to include several $50 \%$ specular reflections. The simulation based on the OSM plasma was then run again. As shown in Fig. 2, the reflections broaden the central $\mathrm{D}_{\alpha}$ emission peak, but not enough to explain the discrepancy with the experimental data. Of course, this change to the simulated diagnostic signal has no effect on the neutral pressure. More realistic treatments of these reflections, including its incorporation into the OSM-EIRENE analysis, will be reported elsewhere.

\section{DISCUSSION}

Ro-vibrational excitation of molecules alters the rates and, hence, the relative importance of molecular and molecular ion reactions [27, 28]. Furthermore, via the "molecular assisted recombination" (MAR) mechanism [27], it can provide an additional source of recombination for deuterium ions. Heretofore, such effects have been largely ignored in divertor simulations. Collisional-radiative models involving vibrationally excited molecules have been assembled $[28,29]$ and compared with experimental data $[27,30,31]$. Additional and higher quality cross-sections are needed to complete these models [28]. Furthermore, the virtually non-existent data on rotationally excited states must be measured or computed and incorporated into them [27]. Because rovibrationally excited states are metastable on typical neutral transport time scales, they must be explicitly transported in codes such as DEGAS 2 and EIRENE. The consequent addition of 17 or more species to the code significantly increases the complexity and computational requirements of these simulations [30].

The importance of MAR to tokamak divertor plasmas remains contentious [27, 30]. Using the rates provided in [28], we estimate the neutral source due to MAR to be $2 \%$ of the total neutral source in the baseline DEGAS 2 simulation and 7\% for the OSM plasma case. This incremental source is too small to explain the discrepancies in neutral pressure and $\mathrm{D}_{\alpha}$ emission.

The structures through which neutral gas escape the divertor to reach the pressure gauges and the main chamber are not toroidally symmetric [5]. In particular, the gaps between the private flux region and the gas box, as well as the divertor bypass and intrinsic leakage pathways, all vary toroidally. Because a net neutral flow is maintained along the neutral pathway, pressure drops exist across these constrictions. The magnitude of the pressure drop is affected by the size of the opening and by the mean free path of the neutral species. The simulated gaps in DEGAS 2, however, are axisymmetric. Without performing three-dimensional simulations, determining the resulting impact on the neutral pressure is difficult. While the error could be significant, we suspect that the 
neutral pressure discrepancy is due to a vastly less subtle effect.

The rapidly evolving picture of scrape-off layer plasma transport may provide just such an effect. Recent observations of filamentary structures [32] and broad main chamber scrape-off layer profiles $[33,34]$ indicate that the "standard" characterization of the scrape-off layer as having diffusive transport and exponentially decaying plasma profiles is qualitatively incomplete. Furthermore, new data and associated analyses indicate that the bulk of the neutrals being recycled into the main chamber are arising from the main chamber walls, not from the divertor surfaces [33, 34], as had hitherto been supposed. The revised description of scrape-off layer behavior as intermittent and non-diffusive [35] invalidates some of the assumptions behind the models used to generate the plasma input to DEGAS 2. Furthermore, the strong $(\sim 100 \%)$ fluctuations reported in these observations imply correspondingly large fluctuations in the physical quantities measured by the diagnostic signals (e.g., the $\mathrm{D}_{\alpha}$ emission profile). The absence of tens-of-kilohertz time resolution in these signals complicates the comparisons with the DEGAS 2 simulations. The origins of the discrepancies reported in this paper may only become clear once our global picture of scrape-off layer transport has been appropriately revised and issues such as diagnostic interpretation have been addressed.

\section{SUMMARY}

We have described neutral transport simulations of Alcator C-Mod experiments. Consistently low neutral pressures and $\mathrm{D}_{\alpha}$ emission profiles qualitatively different from those observed indicate deficiencies in the physics model underlying the code. Aspects of the atomic and surface physics data have been examined as potential areas for future improvement. The surface physics model appears particularly deserving of more careful validation and / or increased realism. The effects of radiation trapping and rovibrationally excited molecules should be incorporated into the simulations; comprehensive and practical descriptions of these processes are needed. The greatest deficiencies in these simulations appear to lie in the models used to tie the experimental data together into the complete two-dimensional plasma profiles input to DEGAS 2. Experimental and theoretical efforts to better understand the plasma transport embodied in these models are currently underway.

\section{ACKNOWLEDGMENTS}

This work was supported by US DOE Contract Nos. DE-AC02-76-CHO-3073 (PPPL) and DE-FC02-99-ER5-4512 (MIT). The authors would also like to acknowledge support from IPP-Jülich, the Alcator C-Mod team, and the University of Toronto.

\section{REFERENCES}

1. ITER Physics Expert Group on Divertor, ITER Physics Expert Group on Divertor Modelling and Database, and ITER Physics Basis Editors, Nucl. Fusion, 39, 2391-2469 (1999). 
2. Tendler, M., and Heifetz, D., Fusion Tech., 11, 289-310 (1987).

3. Feng, Y., Sardei, F., and Kisslinger, J., J. Nucl. Mater, 266-269, 812-818 (1999).

4. Stotler, D. P., Pitcher, C. S., Boswell, C. J., Chung, T. K., LaBombard, B., Lipschultz, B., Terry, J. L., and Kanzleiter, R. J., J. Nucl. Mater., 290-293, 967-971 (2001).

5. Niemczewski, A., Hutchinson, I. H., LaBombard, B., Lipschultz, B., and McCracken, G. M., Nucl. Fusion, 37, 151-163 (1997).

6. Stotler, D. P., and Karney, C. F. F., Contrib. Plasma Phys., 34, 392-397 (1994).

7. Pitcher, C. S., Boswell, C. J., Goetz, J. A., LaBombard, B., Lipschultz, B., Rice, J. E., and Terry, J. L., Phys. Plasmas, 7, 1894-1903 (2000).

8. Pitcher, C. S., Boswell, C. J., Chung, T., Goetz, J. A., LaBombard, B., Lipschultz, B., Rice, J. E., Stotler, D. P., and Terry, J. L., J. Nucl. Mater., 290-293, 812-819 (2001).

9. Lipschultz, B., LaBombard, B., Pitcher, C. S., and Boivin, R., Plasma Phys. Controlled Fusion (2002).

10. Pitcher, C. S., and Stangeby, P. C., Plasma Phys. Controlled Fusion, 39, 779 (1997).

11. Elder, J. D., Lisgo, S., Stangeby, P. C., LaBombard, B., Lipschultz, B., Terry, J. L., Pitcher, C. S., Boswell, C., Porter, G., and Reiter, D., Bull. Am. Phys. Soc., 45, 320 (2000).

12. Biersack, J. P., and Eckstein, W., Appl. Phys., 34, 73 (1984).

13. Weisheit, J. C., J. Phys. B, 8, 2556-2564 (1975).

14. Degas 2 user's manual (2002), http: / /w3 . pppl.gov/degas2/Doc/degas2_all.pdf.

15. Janev, R. K., and Smith, J. J., At. Plasma-Mater. Interaction Data Fus., 4, 1-180 (1993), supplement to the journal Nucl. Fus.

16. Stotler, D. P., Skinner, C. H., Budny, R. V., Ramsey, A. T., Ruzic, D. N., and Turkot, R. B., Jr., Phys. Plasmas, 3, 4084-4094 (1996).

17. Krstic, P. S., and Schultz, D. R., At. Plasma-Mater. Data Fus., 8, 1 (1998), supplement to the journal Nucl. Fus.

18. Kanzleiter, R. J., Stotler, D. P., Karney, C. F. F., and Steiner, D., Phys. Plasmas, 8, 5064-5069 (2000).

19. Reiter, D., et al., J. Nucl. Mater., 241-243, 342 (1997).

20. Haas, G., et al., J. Nucl. Mater., 196-198, 481-484 (1992).

21. Allen, S. L., Rensink, M. E., Hill, D. N., Perkins, D. E., Jackson, G. L., Mahdavi, M. A., and the DIII-D Research Team, J. Nucl. Mater., 162-164, 80-92 (1989).

22. Stotler, D. P., Pigarov, A. Y., Karney, C. F. F., Krasheninnikov, S. I., LaBombard, B., Lipschultz, B., McCracken, G. M., Niemczewski, A., Snipes, J. A., Terry, J. L., and Vesey, R. A., "DEGAS 2 neutral transport modeling of high density, low temperature plasmas," in Proceedings of the Sixteenth International Conference on Fusion Energy, International Atomic Energy Agency, IAEA, Vienna, 1997, vol. 2, pp. 633-639.

23. Boswell, C. J., Terry, J. L., LaBombard, B., Lipschultz, B., and Goetz, J. A., J. Nucl. Mater., 290-293, 556-560 (2001).

24. Fundamenski, W., Stangeby, P. C., and Elder, J. D., J. Nucl. Mater, 266-269, 1045-1050 (1999).

25. Terry, J. L., Lipschultz, B., Bonnin, X., Boswell, C., Krasheninnikov, S. I., Pigarov, A. Y., LaBombard, B., Pappas, D. A., and Scott, H. A., J. Nucl. Mater., 266-269, 30-36 (1999).

26. Scott, H. A., Wan, A. S., Post, D. E., Rensink, M. E., and Rognlien, T. D., J. Nucl. Mater., 266-269, 1247-1251 (1999).

27. Krasheninnikov, S. I., Physica Scripta, T96, 7-15 (2002).

28. Pigarov, A. Y., Physica Scripta, T96, 16-31 (2002).

29. Greenland, P. T., J. Nucl. Mater., 290-293, 615-618 (2001).

30. Fantz, U., Reiter, D., Heger, B., and Coster, D., J. Nucl. Mater., 290-293, 367-373 (2001).

31. Ohno, N., Nishijima, D., Takamura, S., Uesugi, Y., Motoyama, M., Hattori, N., Arakawa, H., Ezumi, N., Krasheninnikov, S., Pigarov, A., and Wenzel, U., Nucl. Fusion, 41, 1055-1065 (2001).

32. Maqueda, R. J., Wurden, G. A., Zweben, S., Roquemore, L., Kugel, H., Johnson, D., Kaye, S., Sabbagh, S., and Maingi, R., Rev. Sci. Instrum., 72, 931-934 (2001).

33. Umansky, M. V., Krasheninnikov, S. I., LaBombard, B., and Terry, J. L., Phys. Plasmas, 5, 33733376 (1998).

34. LaBombard, B., Boivin, R. L., Hughes, J., Lipschultz, B., Mossessian, D., Pitcher, C. S., Terry, J. L., Zweben, S. J., and Alcator Group, Phys. Plasmas, 8, 2107-2117 (2001).

35. D’Ippolito, D. A., Myra, J. R., and Krasheninnikov, S. I., Phys. Plasmas, 9, 222-233 (2002). 


\section{External Distribution}

Plasma Research Laboratory, Australian National University, Australia

Professor I.R. J ones, Flinders University, Australia

Professor J oão Canalle, Instituto de Fisica DEQ/IF - UERJ , Brazil

Mr. Gerson O. Ludwig, Instituto Nacional de Pesquisas, Brazil

Dr. P.H. Sakanaka, Instituto Fisica, Brazil

The Librarian, Culham Laboratory, England

Library, R61, Rutherford Appleton Laboratory, England

Mrs. S.A. Hutchinson, JET Library, England

Professor M.N. Bussac, Ecole Polytechnique, France

Librarian, Max-Planck-Institut für Plasmaphysik, Germany

J olan Moldvai, Reports Library, MTA KFKI-ATKI, Hungary

Dr. P. Kaw, Institute for Plasma Research, India

Ms. P.J . Pathak, Librarian, Insitute for Plasma Research, India

Ms. Clelia De Palo, Associazione EURATOM-ENEA, I taly

Dr. G. Grosso, Instituto di Fisica del Plasma, Italy

Librarian, Naka Fusion Research Establishment, J AERI, J apan

Library, Plasma Physics Laboratory, Kyoto University, J apan

Research Information Center, National Institute for Fusion Science, J apan

Dr. O. Mitarai, Kyushu Tokai University, J apan

Library, Academia Sinica, Institute of Plasma Physics, People's Republic of China

Shih-Tung Tsai, Institute of Physics, Chinese Academy of Sciences, People's Republic of China

Dr. S. Mirnov, TRINITI, Troitsk, Russian Federation, Russia

Dr. V.S. Strelkov, Kurchatov Institute, Russian Federation, Russia

Professor Peter Lukac, Katedra Fyziky Plazmy MFF UK, Mlynska dolina F-2, Komenskeho Univerzita, SK-842 15 Bratislava, Slovakia

Dr. G.S. Lee, Korea Basic Science Institute, South Korea

Mr. Dennis Bruggink, Fusion Library, University of Wisconsin, USA

Institute for Plasma Research, University of Maryland, USA

Librarian, Fusion Energy Division, Oak Ridge National Laboratory, USA

Librarian, Institute of Fusion Studies, University of Texas, USA

Librarian, Magnetic Fusion Program, Lawrence Livermore National Laboratory, USA

Library, General Atomics, USA

Plasma Physics Group, Fusion Energy Research Program, University of California at San Diego, USA

Plasma Physics Library, Columbia University, USA

Alkesh Punjabi, Center for Fusion Research and Training, Hampton University, USA

Dr. W.M. Stacey, Fusion Research Center, Georgia Institute of Technology, USA

Dr. J ohn Willis, U.S. Department of Energy, Office of Fusion Energy Sciences, USA

Mr. Paul H. Wright, Indianapolis, Indiana, USA 
The Princeton Plasma Physics Laboratory is operated by Princeton University under contract with the U.S. Department of Energy.

\author{
Information Services \\ Princeton Plasma Physics Laboratory \\ P.O. Box 451 \\ Princeton, NJ 08543
}

Phone: 609-243-2750

Fax: 609-243-2751

e-mail: pppl_info@pppl.gov

Internet Address: http://www.pppl.gov 